\title{
Welcome to the ACNP 45th Annual Meeting
}

Dear Members and Guests,

It is my pleasure and honor to welcome you to the ACNP's 45th annual meeting. Although the ACNP is engaged in a wide range of activities, our annual meeting remains our jewel. For nearly half a century ACNP members have come together to discuss their most important findings, ask questions, seek knowledge, challenge each other and form new collaborations. This is also a place for old friends to rejoice in each other's company, and new friendships to be born.

The scientific content of this meeting is extraordinary. The richness of the program is a testament to the vibrancy of the College as well as the hard work of the Program Committee. The Committee was overwhelmed with exceptional proposals, and had to make difficult decisions. I am particularly grateful to Larry Siever for chairing the Program Committee, and Robbie Schwarcz his co-chair, for guiding the Committee and ultimately producing so strong and diverse a program. In my initial letter to the membership, I encouraged submissions that elaborated on new targets for drug development and new theories of pathophysiology. I am pleased that the membership responded to this request and the program reflects the elaboration of these themes.
For the last three years Neuropyschopharmacology, our College's official journal, has been publishing the abstracts of the meeting. Consequently these abstracts have been citable and are archival. The decision to move these abstracts from their previously internal audience to the wider archival audience is a decision with far reaching consequences, and thus one that was first taken by ACNP leadership after serious deliberation and only on a pilot basis. We would encourage your input as to whether publication of our abstracts in Neuropyschopharmacology should continue.

We find ourselves in a new venue this year. As our meeting has grown, fewer and fewer sites are able to accommodate all our needs. Our College has prided itself in its encouragement of informality and collegiality in its annual meetings, a goal that is facilitated by accommodations that are consolidated. We are hopeful that our new venue will maximize our comfort and scientific exchange.

I am looking forward to a great meeting. Welcome to Florida.

Kenneth L. Davis, M.D.

President

American College of Neuropsychopharmacology 


\section{Neuropsychopharmacology}

\section{American College of Neuropsychopharmacology 45th Annual Meeting}

\section{ACNP OFFICERS}

President: . . . . . . . . . . . . Kenneth L. Davis, M.D.

President-Elect: . . . . . . . . . . . . William T. Carpenter, Jr., M.D.

Secretary: . . . . . . . . . Alan Frazer, Ph.D.

Treasurer: . . . . . . . . . . A. John Rush, M.D.

\section{ACNP COUNCIL}

Raquel E. Gur, M.D., Ph.D. (04-06)

Mary Jeanne Kreek, M.D. (05-07)

Ellen Frank, Ph.D. (06-08)

Carol A. Tamminga, M.D. (05-06)

Michael Davis, Ph.D. (05-07)

Francine M. Benes, M.D., Ph.D. (06-08)

Peter W. Kalivas, Ph.D. (2006)

Daniel R.Weinberger, M.D. (06-07)

\section{PROGRAM AND SCIENTIFIC COMMUNICATIONS COMMITTEE}

Larry Siever, Chair . . . . . . . . . . . . 2005-07

Robert Schwarcz, Co-Chair . . . . . . . . 2006-08

Lori Altshuler . . . . . . . . . . . . . . . . . 2004-06

Jay Baraban . . . . . . . . . . . . . 2004-06

Jacqueline Crawley . . . . . . . . . . . 2004-06

Suzanne Haber . . . . . . . . . . . . . . . . . . . . 2004-06

Edward Jones . . . . . . . . . . . . . . . . . 2004-06

Edythe London . . . . . . . . . . . . . . 2004-06

Perry Renshaw .....................2004-06

Trey Sunderland . . . . . . . . . . . . . . . . . 2004-06

Karen Berman . . . . . . . . . . . . . . . . . . 2005-07

John Csernansky . . . . . . . . . . . . . . . . . . . 2005-07

Steven DeKosky . . . . . . . . . . . . . . . . . 2005-07

David Goldman . . . . . . . . . . . . . . . . . . . . 2005-07

Dilip Jeste . . . . . . . . . . . . . . . . . 2005-07
Pat Levitt . . . . . . . . . . . . . . . 2005-07

Helen Mayberg . . . . . . . . . . . . . . . . . 2005-07

Lorna Role . . . . . . . . . . . . . . . . . . .2005-07

Gary Small . . . . . . . . . . . . . . . . . . . . . . 2005-07

George Zubenko . . . . . . . . . . . . . . . . . 2005-07

Dennis Charney ... . . . . . . . . . . . 2006-08

Jonathan Davidson ................2006-08

Ariel Deutch . . . . . . . . . . . . . . . . . . 2006-08

Rene Hen ... . . . . . . . . . . . . . . . 2006-08

James Meador-Woodruff . . . . . . . . . . . . . . 2006-08

Ralph Nixon ... . . . . . . . . . . . . . . . . 2006-08

Mary Sano . . . . . . . . . . . . . . . . . . . . 2006-08

Charles France ... . . . . . . . . . . . . . . Ad Hoc

Irwin Lucki .....................Ad Hoc

Raquel Gur ................ Council Liaison

Future ACNP Annual Meetings

December 9 - 13, 2007

December 7 - II, 2008
Boca Raton Resort \& Club

The Fairmont Scottsdale Princess
Boca Raton, Florida

Scottsdale, Arizona 